\title{
Observation and Properties of the van der Waals Dimer Ar...HCCCN Produced in Electrical Discharge
}

\author{
O. Desyatnyk, J. Kosarzewski and Z. KISIEL* \\ Institute of Physics, Polish Academy of Sciences \\ al. Lotników 32/46, 02-668 Warszawa, Poland
}

(Received July 4, 2003)

\begin{abstract}
The weakly bound van der Waals dimer between a molecule of cyanoacetylene and an argon atom was observed by rotational spectroscopy of supersonic expansion. Cyanoacetylene was generated in situ from vinyl cyanide precursor by means of a high voltage discharge just downstream of the expansion nozzle. Both ${ }^{b} R$ - and ${ }^{b} Q$-type rotational transitions were measured and rotational, centrifugal distortion, and nuclear quadrupole splitting constants were determined. Analysis of the data shows that ground state geometry of the dimer is characterised by $R_{\mathrm{cm}}=3.65 \AA$ and $\theta=94.9^{\circ}$, with the Ar atom shifted from the position above the cyanoacetylene centre of mass in the direction of the CN group.
\end{abstract}

PACS numbers: 33.15.Dj, 33.15.Mt, 33.15.Pw, 33.20.Bx, 36.40.Mr

\section{Introduction}

Proliferation of high resolution spectroscopy of supersonic expansion has led to a considerable improvement in the understanding of weak intermolecular interactions. This was achieved by observation and detailed characterisation of many small intermolecular clusters, which is made feasible by the very low effective temperature of the expansion. The most valuable information is obtained by systematical following the properties of judiciously selected families of dimers. For example, studies of van der Waals dimers between a molecule and a rare-gas atom allow insight into the nature of the rather elusive dispersive interaction. One prototype series of such clusters is that between a rare-gas atom and a stick type molecule. Linear dimers of this type are observed when the dispersive inter-

*corresponding author; e-mail: kisiel@ifpan.edu.pl 
molecular energy term is augmented by an appreciable inductive term, otherwise the tendency is to form T-type clusters. The latter series, with members such as $\mathrm{Ar}-\mathrm{CO}_{2}$ [1], Ar-HCCH [2,3], Ar-HCCCCH [4], is of particular interest since it allows monitoring of changes of several factors, especially of the influence of the considerable vibration-rotation effects on dimer properties. Some molecules of interest for such studies are either difficult to handle or to obtain, so that in situ synthesis by electrical discharge or pyrolysis has often been the method of choice for their observation. Cyanoacetylene (ethynyl cyanide, $\mathrm{HC} \equiv \mathrm{C}-\mathrm{C} \equiv \mathrm{N}$ ) is one such species, for which the rotational spectrum has been investigated by synthesis of the molecule in glow electrical discharge [5]. In tests of a newly constructed pulsed discharge nozzle for the Fourier transform microwave spectrometer in Warsaw [6] we used cyanoacetylene as one of test molecules. Cyanoacetylene was in fact obtained in sufficiently large amounts to expect that observation of a dimer with an $\mathrm{Ar}$ atom would be possible. After a short search the dimer Ar...HCCCN was identified at signal to noise sufficient for its accurate characterisation. Details of this investigation are presented below.

\section{Experimental}

Cyanoacetylene was generated from vinyl cyanide (acrylonitryle, $\mathrm{H}_{2} \mathrm{C}=\mathrm{CHC} \equiv \mathrm{N}$ ) precursor by means of a newly constructed pulsed discharge nozzle, shown in Fig. 1. Nozzle design is based on that reported by the Thaddeus group [7], a variant of which was used also in the Kiel laboratory [8]. This nozzle is optimised for observation of stable and metastable products of the discharge reactions and is equipped with a long reaction channel downstream of the discharge region. Our variant is modified in the direction of modular construction allowing easy assembly, cleaning and modification by changing electrode and insulator modules. These are held together by a metal end plate connected by threaded rods directly to the baseplate of the Series 9 General Valve nozzle of the spectrometer. Self-centering and additional insulation are achieved by recessing the electrodes into suitably machined teflon insulators. The electrodes are $3 \mathrm{~mm}$ copper disks and, as in [8], positive polarisation of the high voltage electrode was found to be more efficient, in our case mainly because of less interference with the microwave electronics of the spectrometer. Best results were obtained when argon carrier at 4 atm backing pressure was passed over a test tube containing acrylonitrile at room temperature, and pulsed at $2-3 \mathrm{~Hz}$. The discharge voltage was $800-900 \mathrm{~V}$, and the power supply was connected to the high voltage electrode through a $5 \mathrm{k} \Omega$ series resistor. The nozzle was operated as part of the pulsed supersonic expansion, cavity-Fourier transform microwave (FTMW) spectrometer described in detail in [6]. The spectrometer has most recently been used to investigate the camphor molecule [9], weakly bound trimers $\mathrm{Ar}_{2} \mathrm{HBr}[10]$ and $\left(\mathrm{H}_{2} \mathrm{O}\right)_{2} \mathrm{HBr}$ [11], and is equipped with a novel Stark electrode arrangement for dipole moment measurements [12, 13]. 


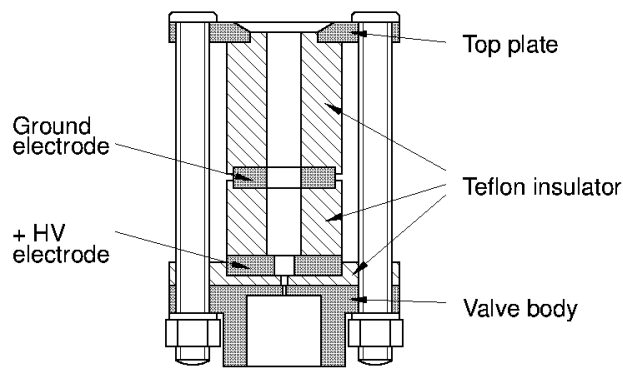

Fig. 1. The modular pulsed discharge nozzle used for observation of the rotational spectrum of Ar..HCCCN by electrical discharge through an acrylonitrile/Ar mixture. The two electrodes are $3 \mathrm{~mm}$ thick copper disks and the expansion diameters are $0.5 \mathrm{~mm}$ in the valve body, $1 \mathrm{~mm}$ in the first insulator, $3 \mathrm{~mm}$ in the high voltage electrode, and $5 \mathrm{~mm}$ downstream of it. The stack of electrode and insulator plates is attached to the baseplate of a standard General Valve Series 9 pulsed valve by four modified $4 \mathrm{~mm}$ diameter screws.

Acrylonitrile was selected as a precursor since the route to cyanoacetylene is by simply stripping off a hydrogen molecule across the CC double bond. In this way it was also possible to avoid using $\mathrm{HCN}$, which is a component of the more traditional HCCH/HCN discharge mixture for producing HCCCN [14, 5]. In fact, our discharge was found to be sufficiently efficient for the emission signal of cyanoacetylene to saturate the detection electronics. A measure of its intensity was that the lines of the three singly substituted ${ }^{13} \mathrm{C}$ isotopomers of cyanoacetylene [15] were observed at intensity corresponding to that of the ${ }^{18} \mathrm{OCS}$ isotopomer in an expansion mixture made up using OCS gas. Thus the signal of the parent isotopomer of cyanoacetylene was estimated to be only 3-4 times weaker than that of OCS. The discharge conditions used for cyanoacetylene allowed observation also of a very large number of other reaction products, such as $\mathrm{HCCNC}, \mathrm{HC}_{5} \mathrm{~N}$, $\mathrm{HC}_{7} \mathrm{~N}$, and $\mathrm{HC}_{9} \mathrm{~N}$, at approximate relative intensities of 170:370:70:10, respectively. The signal of the known weakly bound dimer Ar..HCN [16] resulting from the discharge was also obtained at a level exceeding that of $\mathrm{HC}_{5} \mathrm{~N}$. Rather disappointingly we found that the Stark effect measurements at these conditions were not possible. It turned out that for a given applied electric field the Stark shifts were reduced by at least two orders of magnitude. The most likely explanation is that the large number of charged species produced in the discharge screens the molecules from the external electric field. Attempts to neutralise the expansion by passing it through additional grounded mesh electrodes were not successful.

\section{Rotational spectrum}

The rotational spectrum was searched for using predictions based on T-type geometry discussed below. The dipole moment of the complex is expected to be 
almost completely due to the monomer moment of cyanoacetylene. Since this is at a small angle to the $b$ inertial axis, $\mu_{b}$-type rotational transitions are expected to be dominant, with some possibility for much weaker $\mu_{a}$-allowed transitions. Only $\mu_{b}$-transitions of the complex could in fact be observed, and those were assigned on the basis of the associated nitrogen nuclear quadrupole hyperfine splitting patterns. Altogether 14 different ${ }^{b} R$-type and $6{ }^{b} Q$-type transitions were measured, as summarised in Table I.

TABLE I

The observed $[\mathrm{MHz}]$ and the observed - calculated $[\mathrm{kHz}]$ rotational transition frequencies for Ar...HCCCN.

\begin{tabular}{|c|c|c|c|c|c|c|c|c|c|c|}
\hline$J^{\prime}$ & $K_{-1}^{\prime}$ & $K_{+1}^{\prime}$ & $\leftarrow$ & $J^{\prime \prime}$ & $K_{-1}^{\prime \prime}$ & $K_{+1}^{\prime \prime}$ & $F^{\prime}$ & $F^{\prime \prime}$ & obs. & o. - c. \\
\hline \multirow[t]{3}{*}{1} & 1 & 1 & $\leftarrow$ & 0 & 0 & 0 & 1 & 1 & 5872.3100 & -0.6 \\
\hline & & & & & & & 2 & 1 & 5873.5730 & 2.8 \\
\hline & & & & & & & 0 & 1 & 5875.4600 & 0.2 \\
\hline \multirow[t]{4}{*}{2} & 1 & 2 & $\leftarrow$ & 1 & 0 & 1 & 2 & 1 & 8338.7037 & -0.2 \\
\hline & & & & & & & 2 & 2 & 8339.3147 & -0.2 \\
\hline & & & & & & & 1 & 1 & 8339.7847 & -0.8 \\
\hline & & & & & & & 3 & 2 & 8340.0097 & -0.1 \\
\hline \multirow[t]{5}{*}{2} & 2 & 1 & $\leftarrow$ & 1 & 1 & 0 & 2 & 1 & 15150.6194 & 0.1 \\
\hline & & & & & & & 2 & 2 & 15151.2665 & -1.2 \\
\hline & & & & & & & 1 & 1 & 15151.6370 & -0.7 \\
\hline & & & & & & & 3 & 2 & 15151.9241 & 1.9 \\
\hline & & & & & & & 1 & 0 & 15153.2574 & -1.5 \\
\hline \multirow[t]{5}{*}{2} & 2 & 0 & $\leftarrow$ & 1 & 1 & 1 & 1 & 0 & 15667.9488 & 1.4 \\
\hline & & & & & & & 2 & 2 & 15669.0575 & -1.1 \\
\hline & & & & & & & 3 & 2 & 15669.5612 & 2.3 \\
\hline & & & & & & & 2 & 1 & 15670.3192 & 1.0 \\
\hline & & & & & & & 1 & 1 & 15671.0943 & -2.3 \\
\hline \multirow[t]{2}{*}{3} & 0 & 3 & $\leftarrow$ & 2 & 1 & 2 & 4 & 3 & 6076.9766 & -0.2 \\
\hline & & & & & & & 3 & 2 & 6077.9643 & 1.5 \\
\hline \multirow[t]{5}{*}{3} & 1 & 3 & $\leftarrow$ & 2 & 0 & 2 & 3 & 2 & 10592.2323 & -0.1 \\
\hline & & & & & & & 3 & 3 & 10592.7322 & -0.5 \\
\hline & & & & & & & 2 & 2 & 10593.0394 & 0.5 \\
\hline & & & & & & & 4 & 3 & 10593.3312 & 1.4 \\
\hline & & & & & & & 2 & 1 & 10593.8168 & -0.3 \\
\hline \multirow[t]{3}{*}{3} & 2 & 2 & $\leftarrow$ & 2 & 1 & 1 & 3 & 2 & 17613.7698 & -0.8 \\
\hline & & & & & & & 4 & 3 & 17615.1202 & 0.3 \\
\hline & & & & & & & 2 & 1 & 17615.8700 & 0.4 \\
\hline \multirow[t]{3}{*}{4} & 0 & 4 & - & 3 & 1 & 3 & 3 & 2 & 9261.8134 & 0.5 \\
\hline & & & & & & & 5 & 4 & 9262.0297 & 0.3 \\
\hline & & & & & & & 4 & 3 & 9262.6533 & 0.2 \\
\hline
\end{tabular}


TABLE I (cont.)

\begin{tabular}{|c|c|c|c|c|c|c|c|c|c|c|}
\hline$J^{\prime}$ & $K_{-1}^{\prime}$ & $K_{+1}^{\prime}$ & $\leftarrow$ & $J^{\prime \prime}$ & $K_{-1}^{\prime \prime}$ & $K_{+1}^{\prime \prime}$ & $F^{\prime}$ & $F^{\prime \prime}$ & obs. & o. - c. \\
\hline \multirow[t]{3}{*}{4} & 1 & 4 & $\leftarrow$ & 3 & 0 & 3 & 4 & 3 & 12699.4294 & 0.5 \\
\hline & & & & & & & 4 & 4 & 12699.7153 & -4.7 \\
\hline & & & & & & & 3 & 3 & 12700.1903 & -1.3 \\
\hline \multirow[t]{2}{*}{4} & 1 & 4 & $\leftarrow$ & 3 & 0 & 3 & 5 & 4 & 12700.3285 & 2.0 \\
\hline & & & & & & & 3 & 2 & 12700.5839 & -0.8 \\
\hline \multirow[t]{3}{*}{5} & 0 & 5 & $\leftarrow$ & 4 & 1 & 4 & 4 & 3 & 12341.3830 & -1.4 \\
\hline & & & & & & & 6 & 5 & 12341.4891 & -0.7 \\
\hline & & & & & & & 5 & 4 & 12341.8554 & -0.6 \\
\hline \multirow[t]{3}{*}{5} & 1 & 5 & $\leftarrow$ & 4 & 0 & 4 & 5 & 4 & 14753.4639 & 1.0 \\
\hline & & & & & & & 6 & 5 & 14754.1468 & 1.9 \\
\hline & & & & & & & 4 & 3 & 14754.2870 & 1.2 \\
\hline \multirow[t]{2}{*}{6} & 0 & 6 & $\leftarrow$ & 5 & 1 & 5 & 7 & 6 & 15270.1934 & 2.7 \\
\hline & & & & & & & 6 & 5 & 15270.3732 & -1.7 \\
\hline \multirow[t]{2}{*}{6} & 1 & 6 & $\leftarrow$ & 5 & 0 & 5 & 6 & 5 & 16842.0443 & -2.8 \\
\hline & & & & & & & 7 & 6 & 16842.5288 & 1.6 \\
\hline \multirow[t]{3}{*}{6} & 1 & 5 & $\leftarrow$ & 5 & 2 & 4 & 5 & 4 & 12219.4890 & -2.3 \\
\hline & & & & & & & 7 & 6 & 12219.6474 & 0.7 \\
\hline & & & & & & & 6 & 5 & 12220.6342 & 1.8 \\
\hline \multirow[t]{3}{*}{2} & 2 & 0 & $\leftarrow$ & 2 & 1 & 1 & 2 & 2 & 8867.5266 & 0.1 \\
\hline & & & & & & & 3 & 3 & 8869.3792 & 2.8 \\
\hline & & & & & & & 1 & 1 & 8870.4036 & -0.7 \\
\hline \multirow[t]{2}{*}{2} & 2 & 1 & $\leftarrow$ & 2 & 1 & 2 & 3 & 3 & 10218.0005 & -1.6 \\
\hline & & & & & & & 2 & 2 & 10218.0408 & -1.6 \\
\hline \multirow[t]{3}{*}{3} & 2 & 1 & $\leftarrow$ & 3 & 1 & 2 & 3 & 3 & 8399.7303 & 0.4 \\
\hline & & & & & & & 4 & 4 & 8400.8584 & 1.6 \\
\hline & & & & & & & 2 & 2 & 8401.2499 & -1.5 \\
\hline \multirow[t]{3}{*}{3} & 2 & 2 & $\leftarrow$ & 3 & 1 & 3 & 2 & 2 & 10944.7253 & 0.5 \\
\hline & & & & & & & 4 & 4 & 10944.9346 & 0.5 \\
\hline & & & & & & & 3 & 3 & 10945.5318 & 0.3 \\
\hline \multirow[t]{3}{*}{4} & 2 & 2 & $\leftarrow$ & 4 & 1 & 3 & 4 & 4 & 8015.3145 & -0.2 \\
\hline & & & & & & & 5 & 5 & 8015.9363 & 2.2 \\
\hline & & & & & & & 3 & 3 & 8016.0909 & -2.5 \\
\hline \multirow[t]{3}{*}{4} & 2 & 3 & $\leftarrow$ & 4 & 1 & 4 & 3 & 3 & 11926.1676 & 1.6 \\
\hline & & & & & & & 5 & 5 & 11926.3831 & 0.3 \\
\hline & & & & & & & 4 & 4 & 11927.2264 & -2.0 \\
\hline
\end{tabular}

The transitions of the complex were endowed with the usual Doppler-doubled line shape, as shown in Fig. 2, and the line shape was comparable to that for complexes obtained in conventional manner. The measured transition frequencies were 




Fig. 2. The $b$-type $5_{05} \leftarrow 4_{14}$ rotational transition of Ar $\cdots$ HCCCN observed on electrical discharge through an expanding acrylonitrile/Ar mixture. The displayed spectrum results from averaging time-domain interferograms obtained for 240 gas pulses, each of which was probed with 9 microwave pulses. Two components of the ${ }^{14} \mathrm{~N}$ hyperfine structure are visible, both of which are subject to the Doppler doubling characteristic of unskimmed supersonic expansion.

TABLE II

Spectroscopic constants determined for Ar..HCCCN.

\begin{tabular}{l|c|l|c}
\hline \hline$A[\mathrm{MHz}]$ & $4639.93670(82)^{a}$ & $\Delta_{\mathrm{i}}^{b}\left[\mathrm{uA}^{2}\right]$ & \multicolumn{1}{|c}{$3.66374(9)$} \\
$B[\mathrm{MHz}]$ & $1700.70074(19)$ & $\Delta_{\mathrm{q}}{ }^{c}\left[\mathrm{MHz}^{2}\right]$ & $8.46(12)$ \\
$C[\mathrm{MHz}]$ & $1233.40667(23)$ & & \\
& & $\chi_{a a}[\mathrm{MHz}]$ & $2.0367(11)$ \\
$\Delta_{J}[\mathrm{kHz}]$ & $11.4136(40)$ & $\chi_{b b}[\mathrm{MHz}]$ & $-4.1989(12)$ \\
$\Delta_{J K}[\mathrm{kHz}]$ & $168.65(10)$ & $\chi_{c c}[\mathrm{MHz}]$ & $2.1623(12)$ \\
$\Delta_{K}[\mathrm{kHz}]$ & $-151.34(15)$ & $\mathrm{N}_{\text {lines }}{ }^{d}$ & 65 \\
$\delta_{J}[\mathrm{kHz}]$ & $3.2344(22)$ & $\sigma_{\mathrm{fit}}{ }^{e}[\mathrm{kHz}]$ & 1.48 \\
$\delta_{K}[\mathrm{kHz}]$ & $117.66(12)$ & & \\
$H_{J K}[\mathrm{~Hz}]$ & $-16.6(32)$ & & \\
$H_{K J}[\mathrm{~Hz}]$ & $118.3(93)$ & & \\
\hline
\end{tabular}

${ }^{a}$ The quantities in parentheses are standard errors in units of the least significant digit of the value of the constant.

${ }^{b}$ Inertia defect, $\Delta_{\mathrm{i}}=I_{c}-I_{a}-I_{b}$.

${ }^{c}$ Quartic defect, $\Delta_{\mathrm{q}}=4 C \Delta_{J}-(B-C) \Delta_{J K}-2(2 A+B+C) \delta_{J}$ $+2(B-C) \delta_{K}$.

${ }^{d}$ The number of fitted hyperfine components.

${ }^{e}$ Standard deviation of fit. 
fitted using Pickett's program SPFIT [17] with constants in a standard Hamiltonian, consisting of Watson's $A$-reduced asymmetric rotor term [18] and a single spin nuclear quadrupole splitting term. The resulting parameters are listed in Table II and the overall deviation of fit is seen to be well within the nominal $2 \mathrm{kHz}$ frequency accuracy of the spectrometer.

Two sextic constants were necessary to fit the spectrum to this accuracy and point to a high degree of vibration-rotation contribution from large amplitude motions. Another measure of such contributions are inertial and quartic defects, $\Delta_{\mathrm{i}}$ and $\Delta_{\mathrm{q}}$, which should be zero for a vibrationless planar molecule. Both defects are not dissimilar from those for Ar..HCCCCH [4], for which they are $\Delta_{\mathrm{i}}=3.4162(10) \mathrm{u} \AA^{2}$ and $\Delta_{\mathrm{q}}=4.1(7) \mathrm{MHz}^{2}$. Another point of reference is provided by $\mathrm{Ar}_{2} \mathrm{HBr}$ [10], for which the inertial defect is somewhat greater at $4.5905(12) \mathrm{uA}^{2}$, but the quartic defect of $0.225(11) \mathrm{MHz}^{2}$ is an order of magnitude smaller.

\section{Nuclear quadrupole coupling}

The measured nuclear quadrupole splitting constants for the ${ }^{14} \mathrm{~N}$ nucleus in complexed cyanoacetylene are, in the first approximation, expected to differ from those of the free molecule only due to its different orientation in the inertial frame (cf. Fig. 3). If this were the case then $-2 \chi_{c c}=-4.325(2) \mathrm{MHz}$ in the complex should be comparable to $\chi_{a a}=-4.319(3)$ [14] in cyanoacetylene monomer, and these two values are indeed practically identical. The values of $\chi_{a a}$ and $\chi_{b b}$ in the complex should then be obtainable by rotation of the nuclear quadrupole splitting tensor of the HCCCN molecule to its orientation in the $a b$ plane of the complex. Rotation by the angle $\theta_{z b}=7.83^{\circ}$ between the $b$ inertial axis of the complex and the $C_{\infty}$ axis of cyanoacetylene, leads to $\chi_{a a}=2.039, \chi_{b b}=-4.199$, $\chi_{c c}=2.160 \mathrm{MHz}$. These values are identical with those determined for the complex confirming that there are no appreciable vibrational a veraging effects in play. This degree of rotation also corresponds to $\chi_{a b}=0.874 \mathrm{MHz}$, but in the present case the sensitivity of transition frequencies to this constant is too small for its experimental determination.

\section{Molecular structure}

Determination of reliable molecular geometry for such a floppy dimer is still a challenge, although several useful procedures for minimising the effects of high-amplitude motions on the determined geometry have been developed. Since the rotational spectrum is only of $b$-type character the dimer has to be of T-type geometry, as shown in Fig. 3. It has to be planar, and if it is assumed that the geometry of cyanoacetylene is not changed on complexation then only two structural parameters are to be determined. These are the separation $R_{\mathrm{cm}}$ between the 


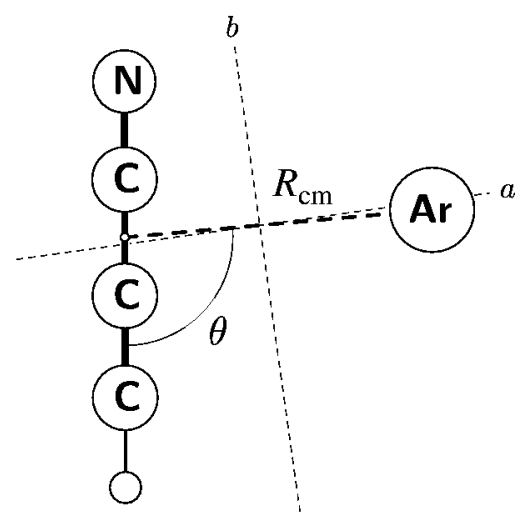

Fig. 3. The geometry of Ar..HCCCN and its orientation in the inertial axes. The centre of mass of cyanoacetylene is almost coincident with the $\mathrm{C}-\mathrm{C}$ bond centre.

Ar atom and centre of mass of cyanoacetylene, and the associated bend angle $\theta$. It has previously been shown [1] that the rotational constant $C$ is to be preferred for determination of $R_{\mathrm{cm}}$ since it is insensitive to effects connected with the angular orientation of cyanoacetylene. For Ar - HCCCN the experimental value of $C$ from Table II and the use of the HCCCN geometry from [19] lead to $R_{\mathrm{cm}}=3.651 \AA$. The angular orientation of the cyanoacetylene molecule was established in the preceding section, and the quadrupolar angle $\theta_{z b}=7.63^{\circ}$ corresponds to $\theta=94.95$ or $85.05^{\circ}$. Because of the symmetry of the inertial ellipsoid it is not possible to distinguish between the two solutions on the basis of inertial information for a single isotopomer. Selection of one of these solutions was made using ab initio calculations, which consistently predicted $\theta>90^{\circ}$, that is Ar atom shifted towards the nitrile group. The MP2/aug-cc-pVDZ level of calculation [20] has been found to be of useful diagnostic value for Ar complexes [10], and for Ar. yields $\theta=95.2^{\circ}$. This direction of shift of the Ar atom is an expected result since it will increase the inductive term between the $\mathrm{Ar}$ atom and the highly polarised $\mathrm{C} \equiv \mathrm{N}$ group. Thus the preferred geometry for $\mathrm{Ar} \cdot \mathrm{HCCCN}$ is $R_{\mathrm{cm}}=3.65 \AA$ and $\theta=94.9^{\circ}$, and both values result from procedures which have been demonstrated to be largely free from vibrational effects. For comparison, the standard $r_{0}$ geometry fitted to all three rotational constants using the programs from the PROSPE website [21] is $R_{\mathrm{cm}}=3.643(11) \AA$ and $\theta=94.2(39)^{\circ}$, where the large errors are the consequence of the large inertia defect.

\section{Conclusions}

Although the use of electric discharge to produce unstable and metastable molecules is well established, the observation of intermolecular complexes of molecules produced in such way is rather less common. In the present investigation we were able to observe, by using a single acrylonitrile precursor, two different 
TABLE III

Comparison of selected properties for several T-type dimers between an Ar atom and a stick-type molecule.

\begin{tabular}{l|c|c|c|c}
\hline \hline & $\mathrm{Ar}-\mathrm{HCCH}^{a}$ & $\mathrm{Ar}-\mathrm{HCCCN}^{b}$ & $\mathrm{Ar}-\mathrm{HCCCCH}^{c}$ & $\mathrm{Ar}-\mathrm{CO}_{2}{ }^{d}$ \\
\hline$R_{\mathrm{cm}}{ }^{e}[\AA]$ & 4.04 & 3.65 & 3.68 & 3.50 \\
$f_{\sigma}\left[\mathrm{Nm}^{-1}\right]$ & 1.05 & 1.55 & 1.64 & 1.74 \\
$\Delta_{\mathrm{i}}\left[\mathrm{u} \AA^{2}\right]$ & 17.97 & 3.66 & 3.42 & 2.89 \\
\hline
\end{tabular}

${ }^{a}$ Ref. [3]. ${ }^{b}$ This work. ${ }^{c}$ Ref. [4]. ${ }^{d}$ Ref. [1]. ${ }^{e}$ Evaluated from the $C$ rotational constant.

complexes between a molecule produced in the discharge and atom of the carrier

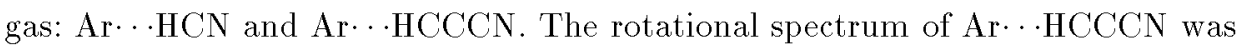
sufficiently intense for its first characterisation. A comparison of some properties of T-type complexes between Ar and stick-type molecules with central carbon atoms is made in Table III.

The stretching constant $f_{\sigma}$ between the Ar atom and the complexed molecule was evaluated from the $\Delta_{J}$ centrifugal distortion constant by using the enhanced diatomic approximation [22]. It can be seen that the complexes conform to the expected inverse proportionality between separation and force constant, i.e. the larger the force constant the smaller the Ar-molecule distance. Nevertheless, since three of the complexes in Table III have a $C_{2}$ symmetry axis and Ar..HCCCN does not, the value of $R_{\mathrm{cm}}$ is not expected to be a very precise indicator of relative binding strength. It can be seen, however, that relative values of $f_{\sigma}$ and of the inertia defect for the four dimers are consistent with binding strength increasing from the left to the right side of Table III. Both $f_{\sigma}$ and $\Delta_{\mathrm{i}}$ indicate

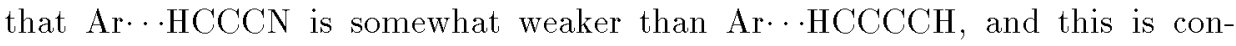
firmed by the MP2/aug-cc-pVDZ binding energies. Application of the counterpoise correction [23] leads to $D_{\mathrm{e}}=190 \mathrm{~cm}^{-1}$ for Ar $\cdots \mathrm{HCCCN}$ and $200 \mathrm{~cm}^{-1}$ for Ar. $\cdot$ HCCCCH. Irrespective of the possible inductive component in the interac-

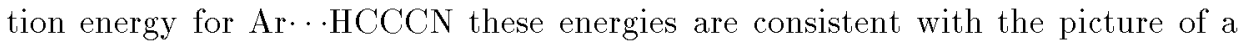
dominant dispersive energy component, as predicted for example by the simple distributed polarisability model [24], in which the atomic polarisability of nitrogen is smaller than that of carbon.

\section{Acknowledgments}

Financial support from the Institute of Physics and from the State Committee for Scientific Research, grant KBN-3T09A-126-17 is gratefully acknowledged.

\section{Note added in proof}

It has just come to the authors' attention that another study of Ar-HCCCN has been completed in the laboratory of Dr. Wolfgang Jäger (Edmonton, Canada), 
to be published in Journal of Chemical Physics. The Edmonton investigation used chemically synthesised HCCCN. The two studies independently reach similar conclusions on the nature of Ar-HCCCN and their results are in many ways complementary.

\section{References}

[1] G.T. Fraser, A.S. Pine, R.D. Suenram, J. Chem. Phys. 88, 6157 (1988).

[2] Y. Ohshima, M. Iiida, Y. Endo, Chem. Phys. Lett. 161, 202 (1989).

[3] T.A. Hu, D.G. Prichard, L.H. Sun, J.S. Muenter, J. Mol. Spectrosc. 153, 486 (1992).

[4] K. Matsumura, Y. Ohshima, Y. Endo, J. Mol. Spectrosc. 185, 178 (1997).

[5] S. Thorwirth, H.S.P. Müller, G. Winnewisser, J. Mol. Spectrosc. 204, 133 (2000).

[6] Z. Kisiel, J. Kosarzewski, L. Pszczółkowski, Acta Phys. Pol. A 92, 507 (1997).

[7] M.J. Travers, W. Chen, J.-U. Grabow, M.C. McCarthy, P. Thaddeus, J. Mol. Spectrosc. 192, 12 (1998).

[8] D.H. Sutter, H. Dreizler, Z. Naturforsch. A 56, 425 (2001).

[9] Z. Kisiel, O. Desyatnyk, E. Białkowska-Jaworska, L. Pszczółkowski, Phys. Chem. Chem. Phys. 5, 820 (2003).

[10] Z. Kisiel, B.A. Pietrewicz, L. Pszczółkowski, J. Chem. Phys. 117, 8248 (2002).

[11] Z. Kisiel, B.A. Pietrewicz, O. Desyatnyk, L. Pszczółkowski, I. Struniewicz, J. Sadlej, J. Chem. Phys., 2003, in press.

[12] Z. Kisiel, J. Kosarzewski, B.A. Pietrewicz, L. Pszczółkowski, Chem. Phys. Lett. 325, 523 (2000)

[13] Z. Kisiel, E. Białkowska-Jaworska, O. Desyatnyk, B.A. Pietrewicz, L. Pszczółkowski, J. Mol. Spectrosc. 208, 113 (2001).

[14] R.A. Cresswell, G. Winnewisser, J. Mol. Spectrosc. 65, 420 (1977).

[15] S. Thorvirth, H.S.P. Müller, G. Winnewisser, Phys. Chem. Chem. Phys. 3, 1236 (2001).

[16] K.R. Leopold, G.T. Fraser, F.J. Lin, D.D. Nelson, Jr., W. Klemperer, J. Chem. Phys. 81, 4922 (1984).

[17] H.M. Pickett, J. Mol. Spectrosc. 148, 371 (1991).

[18] J.K.G. Watson, in: Vibrational Spectra and Structure, Vol. 6, Ed. J.R. Durig, Elsevier, Amsterdam 1977, p. 1.

[19] P. Botschwina, M. Horn, S. Seeger, J. Flügge, Mol. Phys. 78, 191 (1993).

[20] T.H. Dunning, Jr., J. Chem. Phys. 90, 1007 (1989); R.A. Kendall, T.H. Dunning, Jr., R.J. Harrison, ibid. 96, 6796 (1992).

[21] Z. Kisiel, PROSPE - Programs for ROtational SPEctroscopy, available at http://info.ifpan.edu.pl/ ${ }^{\sim} \mathrm{kisiel} /$ prospe.htm.

[22] W.G. Read, E.J. Campbell, G. Henderson, J. Chem. Phys. 78, 3501 (1983).

[23] S.F. Boys, F. Bernardi, Mol. Phys. 19, 553 (1970).

[24] Z. Kisiel, J. Phys. Chem. 95, 7605 (1991). 